Voix et Images

\title{
Gabrielle Roy : de la redondance à l'ellipse ou du corps à la voix
}

\section{Nicole Bourbonnais}

Volume 16, numéro 1 (46), automne 1990

Les correspondants littéraires d'Alfred DesRochers

URI : https://id.erudit.org/iderudit/200876ar

DOI : https://doi.org/10.7202/200876ar

Aller au sommaire du numéro

Éditeur(s)

Université du Québec à Montréal

ISSN

0318-9201 (imprimé)

1705-933X (numérique)

Découvrir la revue

Citer cet article

Bourbonnais, N. (1990). Gabrielle Roy : de la redondance à l'ellipse ou du corps à la voix. Voix et Images, 16(1), 95-109. https://doi.org/10.7202/200876ar d'utilisation que vous pouvez consulter en ligne.

https://apropos.erudit.org/fr/usagers/politique-dutilisation/ 


\title{
Gabrielle Roy: de la redondance à l'ellipse ou du corps à la voix
}

\author{
par Nicole Bourbonnais, Université d'Ottawa
}

Si la description du corps féminin revêt une valeur structurale et symbolique des plus importantes dans Bonheur d'occasion, dans les romans et récits ultérieurs de Gabrielle Roy, elle s'amenuise au point de passer souvent inaperçue. Mais c'est alors de cette rareté même qu'elle tire son sens et une force de frappe d'autant plus grande qu'elle succède à la saturation. $\mathrm{Si}$, dans une précédente étude ${ }^{1}$, c'est le phénomène de la saturation qui a d'abord retenu mon attention, je me propose ici de poursuivre mon examen dans le sens logique de l'évolution vers la rareté.

\section{De la redondance à l'ellipse}

Ce passage de la redondance à l'ellipse s'explique de prime abord par un constat fort simple, celui de la disparition du personnage de gestatrice. Il y a bien la vaillante et grasse Luzina de la Petite Poule d'eau qui, épiphénomène de Rose-Anna, prolonge quelque temps le destin de la génitrice. Toutefois, non seulement les notations relatives au corps porteur sont-elles clairsemées, mais déjà l'accent se déplace vers la légèreté et la mobilité corporelle pour en oublier la masse et le poids. En effet, les grossesses quasi annuelles de Luzina deviennent l'occasion d'un voyage périodique à Sainte-Rose-du-Lac qui, dans cette existence toujours uniforme, [...] était la grande, l'unique aventure ${ }^{2}$. Comme nous le verrons plus loin, ce personnage s'inscrit bien davantage dans le paradigme du déplacement que dans celui de la reproduction. On remarque encore de rares figures épisodiques comme celle de madame Pasquier au ventre gonflé, qui, dans sa terrible tâche de pourvoyeuse de l'espèce, reste couchée presque tout au long de sa grossesse ${ }^{3}$. Mais ce ne sont là que de brèves mentions qui sillonnent le texte le temps d'un éclair et qui fonctionnent comme un acte de commémoration de l'origine,

1 «Gabrielle Roy: la représentation du corps féminin», Voix \& images, vol. XIV, $n^{\circ} 1$ (40), automne 1988 , p. $72-89$.

2 Gabrielle Roy, la Petite Poule d'eau, Montréal, Beauchemin, 1964, p. 15.

3 Gabrielle Roy, Cés enfants de ma vie, Montréal, Staǹké, 1977, p. 117, 121 et 115 respectivement (désormais : EV). 
ressuscitant avec force et acuité le personnage exemplaire de RoseAnna. De la sorte, en choisissant de renvoyer dans une mince lisière d'ombre le corps reproducteur qu'elle fit naguère surgir en pleine clarté, Gabrielle Roy ne le voue pas à l'oubli, mais le signale plutôt comme gravé à jamais dans la mémoire collective. Un simple rappel suffit à dire à quel point ce corps est périmé et non viable. Tel ce gros paquet de jupes, de laine, de châles ${ }^{4}$, qui a pour nom Thérèse Veilleux et qui ne quittera la pénombre de sa chambre de perpétuelle accouchée que trop tard.

Du côté des futures gestatrices, l'impossibilité est aussi grande. Comment, dans le contexte social de l'époque décrite, mettre en scène, dans la vraisemblance et la crédibilité, un jeune personnage féminin à la chair rayonnante qui, arrivant à se soustraire à son "destin biologique" ou à la perdition, connaitrait le droit à la jouissance et réaliserait en toute liberté des idéaux autres que le service et le sacrifice? À l'échafaudage tout imaginaire d'une fiction de l'avenir, l'auteure préfêre la mise en déroute d'un présent inacceptable.

Le cas de l'Esquimaude, Elsa, est à cet égard exemplaire. Avec ce personnage de jeune fille, doublement aliénée par son sexe et par sa race, Gabrielle Roy reprend le modele de Florentine mais comme en accéléré et dans une surexposition éclatante. Si, dans Bonheur d'occasion, le viol de Florentine par Jean se dissimule sous le masque de la séduction, dans la Rivière sans repos, tout maquillage est banni au profit d'un éclairage brutal. Voici le scénario: la jeune Elsa à la chair dodue - ici l'embonpoint tant souhaité par Jean, à l'appétit d'ogre, est actualisé - se trouve', dès le début du récit, entraînée malgré elle derrière les buissons par un soldat américain, sans nom et sans visage, qui la viole dans l'ombre, le silence et la plus grande hâte. Il n'a même pas succombé à ses charmes car Elsa, est-il dit, n'a rien pour attirer un blanc: ce qui est crûment exhibé ici, c'est le désir mâle à l'état brut, qui ne vise que son assouvissement sans une seule pensée pour l'" objet" utilisé. D'autre part, jamais victime plus docile et plus consentante, semble-t-il, n'a existé. Cette scène de viol, tragique en soi, frôle presque la caricature tellement est grande la volonté de l'auteure de rendre manifeste la confusion totale qui règne dans l'esprit d'Elsa contradictoirement sollicité par l'univers de rêve que proposent les films conçus pour des Blancs et les valeurs plus terre-à-terre que lui offre la réalité de sa vie d'Inuit:

Hors cette panique [celle du garçon], tout était cependant tel qu'au cinémá, étrange, lointain, à peine vraisemblable. [...]

Le G.I. poussa Elsa. Il s'abattit sur elle. [...] 


\begin{abstract}
Même les parents d'Elsa n'allaient pas plus vite. C'est peutêtre pourquoi elle était stupéfaite au point de séparer mal la réalité du buisson, de l'histoire d'amour vue à l'écran plus tôt dans la soirée et dont ceci pouvait sembler la suite. ${ }^{5}$
\end{abstract}

L'opposition rêve-réalité pouvait se résoudre par le mariage pour une Florentine également victime d'un code culturel artificiel. Rien de tel pour Elsa qui non seulement n'arrive pas à identifier l'agresseur mais non plus à le condamner pour un acte jugé naturel selon le code des siens (s'il est accompli librement, il va sans dire). Et puis n'est-il pas un Blanc, par définition un être supérieur? Propulsée bien malgré elle dans la maternité, après s'être soumise au géniteur, elle devient l'esclave du fils, un petit Blanc qu'elle idolâtre. Elle finira, en dépit de sa volonté - n'était-ce pas aussi la crainte de Florentine? - , par ressembler à sa mère dans sa chair délabrée, dans une sorte de honte de l'âme ${ }^{6}$. Tout se passe comme si était donnée au lecteur une version non embellie du sort qui aurait pu être réservé à Florentine. Il a suffi à Elsa d'avoir un corps de femme et d'être de la race des Inuit pour que le cours de son existence soit à jamais fixé. Pas de rachat possible pour " elle» qui sombre dans l'indifférence et la folie.

Outre cette réplique navrante et caricaturale d'elle-même, Florentine n'aura comme épigones que de rares fantômes en qui se reconnaissent des bribes éparses de son corps asservi. Ce sera par exemple Nina, la petite serveuse de la Montagne secrète, en qui les clients de l'auberge voient un corps jeune et désirable tandis que la vue de son visage, fin et délicat, quoïque crispé, inspire à l'artiste Pierre Cadorai une réflexion qui dépasse le cas particulier pour rejoindre l'universelle condition féminine:

$\grave{A}$ travers ce visage, le sort féminin lui sembla tout à coup pathétique au delà de tout. [...] Elle lui apparaissait comme une petite Ėve d'une sorte nouvelle au milieu de ces hommes rudes - une Ėve qui déjà cependant avait une amère connaissance de la vie. 7

Il y a aussi le personnage de la jeune institutrice qui acquiert, l'espace d'un moment, dans le regard de convoitise de l'autre sexe, des attraits physiques propres à servir d'appât. Le lecteur de la nouvelle intitulée "De la truite dans l'eau glacée" se souviendra que le père du jeune Médéric invite l'institutrice à dîner dans le but évident d'encourager une idylle entre elle et son fils. Il la déclare jolie à ravir (EV, p. 172). Sur le chemin du retour, alors que Médéric la ramène

5. La Rivière sans repos, Montréal, Librairie Beauchemin limitce, 1970, p. 126127.

$6 \quad$ Ibid., p. 305.

7 La Montagne secrète, roman, Montréal, Librairie Beauchemin limitée, 1962, p. 34 . 
dans la grande berline et qu'elle est tentée par l'aventure dans ce qu'elle a de romanesque et d'inédit, se produit une scène d'autocontemplation très semblable à celles qui campent une Florentine aliénée dans Bonheur d'occasion:

La vitre assombrie me renvoya le reflet de mon visage. Il m'apparut flou, gracieux, avec de lointains yeux qui perçaient la neige en tourbillons et des cheveux fous qui moussaient. Je ne pouvais en détacher le regard. (EV, p. 183)

On retrouve les motifs qui désignent le rêve et la perte de l'identité: le flou, les yeux lointains, les cheveux mousseux, la neige tourbillonnante, etc. Mais, cette fois, la noyade dans le reflet envoûtant de la glace n'aura pas lieu: la jeune institutrice, dans une conscience claire de soi, échappe à l'envoûtement maléfique. Il ne sera dès lors plus question de ses charmes physiques. La même lucidité sera octroyée à l'adolescente de Rue Deschambault qui, brièvement attirée par ces artifices et déguisements féminins que sont les bijoux, le fard, les talons hauts, retrouvera son naturel et ses esprits lorsqu'elle surprendra le discours maternel suivant:

Au fond, il n'y a pas d'égalité entre les hommes et les femmes. Les belles vertus: la loyauté, la franchise, la droiture, l'admirable simplicité, vous les revendiquez pour vous alors que vous prisez les femmes pour leurs détours, leurs caprices. C'est très mal... (RD, p. 239)

Participant d'une autre part de Florentine, sa maigreur, quelques personnages de jeunes filles seront fragiles, évanescents, destinés à inexister. Trois des sœurs de la narratrice dans Rue Deschambault ont pour tâche de représenter celles qui fuient hors de la matière. Pour Agnès, Alicia et Odette, ni mariage ni procréation ne sont en vue mais ni, non plus, épanouissement ou corps euphorique. Le portrait très stylisé d'Alicia fait penser à un tableau en noir et blanc, tout en contrastes. La chevelure d'un noir profond s'oppose à son fin visage pâle (RD, p. 165), à sa longue robe blanche (RD, p. 167) qui, à leur tour, forment un contraste avec les beaux sourcils [...] si fortement arqués, si hauts et purs (RD, p. 165). L'impossible réconciliation de l'esprit et de la matière entraîne Alicia dáns l'abîme de la folie: elle est comme brisée, brisée (RD, p. 177). Cette irréductible division est de nouveau illustrée dans le portrait des deux autres sœurs. À Agnès, aux maux de tête fréquents, est réservée la part de Marthe; elle se donne aux siens, vivant par procuration dans l'orbite parentale. Odette, la princesse au cœur porté à la mystique (RD, p. 69), n'accepte pas de se soumettre aux travaux ménagers. Elle n'a donc pas droit à un seul énoncé corporel mais, en revanche, elle est gratifiée d'un luxe de détails concernant les toilettes recherchées qui lui permettent de masquer le corps honni et d'être emportée, en imagination, loin du traditionnel centre de gravité féminin: 
Je me rappelle les jupes d'alpaga qu'elle portait presque à la cheville; on apercevait ses belles bottines montantes dont le bout était pointu et retourné un peu en quille de bateau; il fallait un crochet spécial pour les dix petits boutons noirs. Odette avait aussi des corsages tout bouffonants avec de grands cols dits matelots, d'une soie si fine qu'elle devait mettre par-dessus un cache-corset, mais comme celui-ci était également fin, Odette devait porter sous le cache-corset autre chose encore. Pourtant, ce qu'elle avait de plus beau selon moi, c'était sa toilette pour aller en auto avec son amie de cour, Carmel, qui était fille de parents riches. Odette enfilait un grand manteau raglan, de couleur beige - et déjà elle prenait une allure d'exploratrice - des verres fumés et une espèce de casque à visière, duquel descendait un ample voile qui était destiné à la protéger contre la poussière de la route. Ainsi habillée, elle allait parfois en auto avec Carmel jusqu'à dix milles au loin... (RD, p. 69-70)

Exilé, le corps s'estompe, inimaginable sous le défilé de costumes étranges, romanesques, qui visent l'évasion. L'ultime travestissement, le seul possible, sera celui du voile de la religieuse. Par ailleurs, le seul personnage de jeune fille de Rue Deschambault à ne pas être privé de signes corporels dénotant vigueur et santé est celui de Georgiana, aux deux fortes tresses noires roulées et attachées avec un ruban au-dessus des oreilles, aux yeux excessivement parlants, toujours prête à éclater de rire (RD, p. 58). Or elle est aussi la seule à courir aveuglément au mariage, c'est-à-dire au malheur. Quand il s'agit de la femme, le corps ne peut être contourné: nié, il empêche de vivre, reconnu, il réalise le triste sort de la mal-mariée.

Progressivement, dans ces récits écrits sur le mode autobiographique, figures passagères et rapides esquisses de destins avortés finissent par composer un effet symbolique d'autant plus efficace qu'il s'inscrit dans le sillage réaliste du premier roman qui sert de passé et de mémoire à l'œuvre entière. En délaissant le lexique et les figures du corps-objet, Gabrielle Roy transforme l'acte illocutoire de Bonheur d'occasion en acte performatif. Comme si, dans un geste unique et exemplaire, elle avait liquidé en une fois la sauvagerie sociale qui consiste à enfermer la femme dans la jungle du corps. Désormais, par un appel suggestif au mimétisme magique, elle élimine du plan romanesque ce qui n'est pas viable sur le plan existentiel.

\section{Un fructueux échange}

Pourtant, ce qui se réalise dans l'œuvre royenne, c'est moins une éviction radicale du corps ancillaire qu'un transfert de ses forces vitales. Un échange s'opère en vertu duquel la perte du corps, ou plutôt son dépassement, donne accès à un extraordinaire pouvoir 
d'invention et de création. Gabrielle Roy ne cherche pas, à l'instar de Colette, à créer des personnages féminins affranchis, aux gestes virils et hardis, dotés d'une saine sensualité, se mouvant avec aisance dans la plus grande autonomie d'esprit et de corps. Mais plutôt, de même que Colette dans la Naissance du jour, elle choisit de peindre la femme âgée qui, devenue inapte à la séduction comme à la reproduction, peut enfin échapper à l'opprimante différenciation sexuelle.

Un survol rapide des protagonistes féminines des récits postérieurs à 1945 permet de constater que la femme vieillissante y occupe une place de choix, tandis que mères fécondes et jeunes filles en fleurs n'obtiennent plus qu'un rôle épisodique et secondaire. Dans Rue Deschambault, Éveline, la mère de la narratrice, a dépassé la période de fécondité: Christine, la dernière-née, témoigne de ce terme assigné à la saison productive. Dans la première nouvelle de la Route d'Altamont, intitulée "Ma grand-mère toute-puissante", Éveline, qui descend du tram accompagnée de sa mère, apparaît aux yeux de la petite comme une assez vieille personne en aidant une autre beaucoup plus vieille ${ }^{8}$. La grand-mère est en effet devenue une grande vieille, à la vue affaiblie, à l'ouïe défectueuse (RA, p. 13). Même scénario pour Martha, la protagoniste si attachante du "Jardin au bout du monde", qui, ses enfants partis, est désormais vieille, brisée, presque morte en vérité 9 .

Quant à Luzina, bien que toujours féconde, elle peut être considérée comme un personnage de transition, une mère en voie de déplacement. N'est-ce pas grâce à elle que l'institutrice fait son entrée dans l'univers maternel de reproduction de la matière qu'elle transmute en monde géniteur des esprits? Par ses fréquentes sorties hors des frontières familières; par son acharnement à ouvrir son univers fermé aux étrangers, déténteurs du savoir, et même par cette incroyable émigration vers l'île sauvage et isolée de la Petite Poule d'eau, le personnage de Luzina s'inscrit bien davantage dans le paradigme de la transcendance que dans celui de l'enlisement dans la chair. De plus, contrairement à ce qui se passe pour Rose-Anna, son corps est sommairement décrit. Un minimum d'éléments descriptifs suffisent à placer le corps, ce qui autorise un passage rapide vers la zone de la pensée et de la création. Une relation inversement proportionnelle semble s'établir entre le degré de présence corporelle et l'émergence de la pensée propre: moins pèse le fardeau du corps, plus l'esprit allégé est libre de s'envoler vers l'infini.

D'où l'importance grandissante du déplacement dans l'univers diégétique comme métaphore de la liberté et comme tenant-lieu de

8 La Route d'Altamont, roman, Montréal, Éditions HMH, 1966, p. 44 (désormais: RA).

9 Un jardin au bout du monde et autres nouvelles, Montréal, Librairie Beauchemin limitée, 1975, p. 211 (désormais: JM). 
corps. Après la mobilité si prégnante de Luzina, ce sera, dans le récit "Les déserteuses", l'irrépressible désir d'Éveline d'accomplir le long trajet vers le Québec et l'enfance qui témoignera de cette délivrance corporelle. Ou dans le récit éponyme du recueil intitulé De quoi t'ennuies-tu, Éveline ? ${ }^{10}$, le même personnage vieilli entreprend un long et merveilleux voyage en autobus qui la conduit du Manitoba vers la Californie et dont l'enchantement lui fait oublier la mort de son frère Majorique. Citons encore le cas de ce personnage quasi mythique qu'est la cousine Martine, vieille petite mère humaine, qui, en dépit de son ventre creux et de son petit corps fluet ${ }^{11}$, a réussi à méttre au monde quatorze enfants à la taille gigantesque. Or le récit qui lui est consacré relate, non pas son interminable tâche d'accoucheuse, mais bien cet exploit que représente pour cette menue vieille, déformée par l'arthrite, la pénible descente vers le fleuve SaintLaurent, symbole de mouvement, de puissance et d'éternité.

Enfin soustraites à leur corps, les "vieilles" ne tiennent plus en place, elles vont et viennent, à la poursuite de l'inconnu ou, tout simplement, de leurs désirs. La mère de la Route d'Altamont, pourtant vieille et parfois lasse (RA, p. 199), à la vue des collines, se mit à grimper, alerte encore, avec des mouvements de chevrette, la tête d'instant en instant levée vers le haut (RA, p. 205). Et paradoxalement, la mort s'éloigne d'elle, qui est vieille et lasse, tandis que pour les plus jeunes, telles Florentine et Rose-Anna, la mort s'insinue déjà dans leur corps maigre et cadavérique. Procédant à l'envers de la démarche accumulatrice de Bonheur d'occasion dans une sorte de démonstration a contrario, Gabrielle Roy établit la preuve que la vraie nature de la femme - celle qui fait retour une fois le joug social disparu - la porte vers les grands espaces et le grand large. La prédilection accordée au regard, au détriment du reste du corps, en témoigne encore. Vieille mère, grand-mère et autres vieilles de passage possèdent un regard intense, aigu, curieux, qui ne cesse de se promener des êtres aux choses de la nature: yeux fins, rusés et clairvoyants (RA, p. 31) de la grand-mère ou, seuls visibles au fond du visage (JM, p. 204), les yeux de Martha fixant les couleurs vives du ciel.

Mais encore plus que le déplacement physique, ce qu'autorise l'effacement du corps, c'est l'apparition d'un fertile esprit d'invention. Alors que Rose-Anna et Florentine s'embourbent dans des tâches répétitives et improductives, les vieilles mères, laissant libre cours à leur dynamisme et à leur énergie, s'occupent à recréer le monde. Leur cuvre, dès lors, s'apparente à celle du Créateur. Pour donner à

10 De quoi t'ennuies-tu, Éveline? suivi de Ely! Ely! Ely! Récits, Montréal Boréal Express, 1984.

11 "Le jour où Martine descendit au fleuve", Cet été qui chantait, Montréal et Québec, les Éditions françaises, 1972, p. 152 et 153. 
entendre la puissance créatrice de la femme, l'auteure a recours à la métaphore divine. À sa grand-mère toute-puissante qui transforme chiffons et boutons en une ravissante poupée, la petite Christine déclare: Tu es Dieu le Père. Tu es Dieu le Père. Toi aussi, tu sais faire tout de rien. (RA, p. 28) Autre femme-dieu que Martha de Volhyn qui, au sein de la désespérante immensité des plaines, image du vide et de l'indifférence, fait surgir avec quelques pauvres graines, de presque rien, un petit jardin éclatant de fleurs et de couleurs, sorte de paradis terrestre qui communique la joie et le désir de vivre à ceux qui le découvrent ${ }^{12}$. Le talent de conteuse de la mère dans Rue Deschambault s'apparente aussi au pouvoir démiurgique, alors qu'elle recrée avec tant d'art le voyage au Québec qu'elle réussit à détourner la colère du père, dressé contre les déserteuses. Constamment, le régime imaginaire que l'auteure déploie autour de la constellation mère-dieu déplace le corps, passif récepteur de la semence, vers un fonds matriciel actif et créateur.

Si l'équation mère-dieu met en valeur la dynamique créatrice de la femme âgée, par un mouvement inverse, l'analogie père-dieu fait ressortir les attributs menaçants de la toute-puissance divine. Dans un intéressant jeu de bascule, l'image d'un père irascible et négateur vient appuyer, en s'y opposant, la figure d'une mère orientée vers les forces vives de la vie. L'époux de Martha, le sauvage Stéphan, muré dans un silence rageur, laissant sa ferme à l'abandon, conçoit l'Être suprême comme un tyran en quête de délations et de châtiments. Autre cas exemplaire que celui du père dans Rue Deschambault qui, par son métier d'agent d'immigration, est amené à établir les immigrés sur des terres et à régir leur destinée. Cela s'avère tout particulièrement avec les Ruthéniens, qu'il installe sur des terres abandonnées près de la rivière Perdue, au cours asséché. En peu de temps, grâce à leur dur labeur, ils pourront habiter un lieu édénique où la rivière coule à nouveau. Devant ce "miracle", les Petits-Ruthènes vouent à leur père d'adoption un culte égal à celui de Dieu. Or, lorsqu'un soir un incendie ravage leurs terres et leurs biens, le père accuse ses protégés d'avoir soulevé la colère de Dieu par leur orgueil, ce qui pousse le chef, Jan Sibulesky, à se lancer dans une maison en flammes, l'icône de la Vierge à la main, dans le but d'apaiser la vengeance divine. Le père ne se pardonnera jamais son grand tort, qui avait été d'interpréter Dieu aux hommes (RD, p. 161). Son grand tort, évidemment, avait été de parler de la colère de Dieu. (RD, p. 156) Est ici évident le parallèle établi avec la chute d'Adam, chassé du paradis terrestre pour sa désobéissance et son orgueil, et condamné à la souffrance et à la mort. Dans le partage des attributs divins, la toute-puissance écrasante semble réservée au père alors qu'à la mère revient la puissance créatrice.

12 Voir mon article intitulé "La symbolique de l'espace dans les récits de Gabrielle Roy", Voix \& images, vol. VII, ${ }^{\circ} 2$, hiver 1982, p. 367-384. 


\section{Une transmission matrilinéaire}

Cette mise en scène d'un esprit géniteur propre aux femmes libérées de leur corps est marquée par une circonstance narrative bien déterminée et récurrente: l'acte créateur a toujours lieu devant le même témoin privilégié qui en reçoit sur-le-champ les effets salutaires. Il s'agit du personnage autobiographique de Christine, enfant ou adulte, institutrice ou narratrice. Tantôt, c'est la capacité d'invention de la grandmère qui a pour effet de tirer la petite Christine de son ennui pour l'entraîner dans un espace ludique propice à l'essor de l'imagination: J'observai qu'elle commençait à m'associer à son œuvre créatrice, et je fus encore plus fière de ses talents. (RA, p. 22) Tantôt c'est la voix conteuse $^{13}$ de la mère qu'écoute Christine, émerveillée, attentive à bien saisir la recréation des souvenirs comme des oiseaux en plein vol (RD, p. 138). Le même sens symbolique émerge avec force dans "La route d'Altamont " alors que la mère, retrouvant ses collines perdues, montre à sa fille la puissance de la rêverie poétique et le chemin du retour vers l'enfance, source inépuisable de récits. D'ailleurs, depuis son arrivée au Manitoba, n'a-t-elle pas inlassablement repris le récit de l'émigration de ses parents, sorte de canevas où elle avait travaillé toute sa vie comme on travaille une tapisserie, nouant des fils, illustrant tel destin (RA, p. 214)? Si à l'une appartiennent l'espace et la transmission orale, l'autre revendique le temps et l'écrit: en effet, la fille retrouve une première fois les chères collines perdues de la mère, puis les perd de nouveau, ce qui lui permettra de les recréer, leur conférant ainsi l'éternité. Car, toute créatrice soit-elle, la vieille mère possède un corps éphémère qui se dégrade, qui lui fait défaut. Elle est vouée à la mort comme en témoigne le dénouement chaque fois retenu par l'auteure tant pour Martha que pour Éveline et la grand-mère.

La relation insistanté qu'établit l'œuvre royenne entre, d'une part, le complexe des vieilles mères originales et originelles et, d'autre part, le personnage de l'observatrice en posture d'apprentie inscrit la filiation mère-fille dans l'axe de la transmission du souffle créateur, tandis qu'est bloqué l'automatisme de reproduction de l'espèce. Transmission matrilinéaire de l'origine qui, grâce à la voix de l'écrivaine prenant le relais du corps maternel, assure une nouvelle descendance de l'esprit. C'est ainsi que dans le récit intitulé «Un jardin au bout du monde", le témoin privilégié n'est nul autre que le personnage de l'écrivaine elle-même, qui, en proie à un profond découragement, recevra les effluves bénéfiques du petit paradis terrestre créé par Martha: En ce temps-là, souvent je me disais : à quoi bon ceci, à quoi bon cela? Écrire

13 René Payant, "L’impossible voix ", Marguerite Duras à Montréal, textes réunis et présentés par Suzanne Lamy et André Roy, Montréal, Éditions Spirale, 1981, •p. 169. 
m'était une fatigue. (JM, p. 155) Mais le spectacle inédit d'une masse de couleurs [celles des fleurs] ardentes, au plus creux de la désolation et de la sécheresse (JM, p. 155), opère en elle un bouleversement tel qu'elle se remettra à l'écriture afin d'immortaliser le nom et l'histoire de Marie Martha Yaramko, de l'empêcher de sombrer dans le néant et l'oubli. De l'union avec la mère tutélaire et rédemptrice est née cette voix.intemporelle qui insémine le texte, donnant naissance, à son tour, à une figure maternelle mythique et magnifiée.

\section{Du corps à la voix}

Le véritable dédouanement du corps ne s'effectue donc qu'avec l'arrivée et l'établissement d'une voix expressive qui domine toutes les autres, celle de la narratrice écrivaine. Avec le choix narratif de la première personne, apparue dès Rúue Deschambault, naît un person-nage duel dont une moitié a pour mission d'observer et de commenter les faits et gestes de l'autre moitié, chargée de servir d'exemple. Séparation temporelle certes, où l'une, enfant, adolescente, institutrice, est toujours rejetée sur la grève du passé tandis que l'autre flotte, adulte achevée, immuable et intemporelle. Séparation d'ordre spatial aussi dans la mesure où celle qui est l'objet de la narration est liée à son corps et à la terre tandis que l'autre, sujet de la narration, erre librement, voix désincarnée, invisible et omniprésente.

Tout semble indiquer que l'évolution du personnage de Christine obéit à une loi de l'écriture de Gabrielle Roy qui commande l'effacement progressif du corps féminin. En tant qu'enfant, elle est dépeinte comme la petite chétive (RA, p. 10), celle qui a un petit air abattu et maigrichon (RA, p. 79), qui est chétive et maigriotte (RA, p. 95). Loin de l'entraver, ce corps maladif la libère pour les jeux de l'imagination qui requièrent la souplesse du corps comme celle de l'esprit. Montée sur des patins à roulettes ou sur des échasses, la fillette s'élance au loin, en pays étranger (RA, p. 64), ou vers les montagnes Rocheuses. Elle s'imagine être La Vérendrye, le plus grand explorateur du Canada (RA, p. 65). Clouée sur son hamac par la coqueluche, elle s'évade par l'esprit dans des régions oniriques suscitées par le tintement du carillon de verre, léger, aérien. C'est encore cette fragilité corporelle - elle est dite frêle de santé (RD, p. 37) - qui pousse le père à la surnommer "Petite Misère", nom qu'elle exècre, le reconnaissant d'instinct comme le signe de son identification à la fonction maternelle. "Mère " et "misère " sont synonymes dans l'œuvre royenne, comme l'a bien vu André Brochu à propos de Bonheur d'occasion:

\section{[...] Rose-Anna est l'incarnation même de la mère misé- rable. ${ }^{14}$}

14 André Brochu, "Thèmes et structures dans Bonheur d'occasion", l'Instance critique, 1961-1973, Montréal, Lemćac, 1974, p. 229. 
[...] Florentine représente vraiment pour le jeune homme, orphelin depuis sa plus tendre enfance, la mère - en même temps que la misère. ${ }^{5}$

L'enfant chétive ne connaîtra pas la transformation rituelle en objet narcissique et séducteur. Tant à l'adolescence que plus tard, est maintenu, comme on l'a vu, le déni du corps. Ou sa présence est négligeable, ou elle n'est pas signalée, ou elle est disqualifiée comme mensongère et importune. Dès sa première entrée en fonction, le corps de l'institutrice est jugé incompatible avec sa tâche difficile et exigeante:

La dame chez qui j'allais loger dit en me voyant:

- Hein! Ce n'est pas vous la mâttresse d'ecole! Oh non, ce n'est pas possible! Elle ajusta ses lunettes pour mieux me voir. Mais ils ne vont faire qu'une bouchée de vous. (RD, p. 284)

Si la dévoration n'a pas lieu, c'est que la finesse et l'astuce sont décuplées pour suppléer le corps diminutif. Ce manque de robustesse et de chair n'est pas sans rapport avec la décision de quitter l'enseignement. Comme la "vocation " maternelle, celle de l'institutrice est indissociable du corps, c'est-à-dire du destin féminin qui est celui de la reproduction. Car n'y a-t-il pas coïncidence de but et de moyen entre ce qui conduit, dans le dévouement, la patience et l'oubli de soi, à la reproduction des corps comme à celle des mentalités? N'est-ce pas le même asservissement qui est dénoncé, au nom de la mère, dans Bonheur d'occasion, et, au nom de l'institutrice, dans Ces enfants de ma vie?

Si jeune, je me voyais enfermée pour la vie dans ma tâche d'institutrice. (EV, p. 147-148)

L'effroi que j'avais éprouvé un jour de devoir rester toute ma vie enchaînée à ma tâche d'institutrice me permettait d'entrevoir ce que pouvait être son sentiment [celui de la mère] à la perspective de ne plus quitter sa place de prisonnière, à la fenêtre. (EV, p. 48) ${ }^{16}$

Est-ce un hasard si c'est la mère qui est choisie comme terme de comparaison, comme prisonnière? La prison étant, bien entendu, celle du corps: la mère, à la hanche fracturée, n'arrive plus à marcher. Il ne s'agit pas de nier l'estime et le respect que l'auteure manifeste dans ses œuvres pour la fonction de l'institutrice, certes valorisée ainsi que l'écrit Gabrielle Pascal:

\footnotetext{
15 Ibid., p. 238.
}

16 Autre citation éclairante: Je me voyais dans vingt ans, dans trente ans, à la même place toujours, usée par la tâche, l'image même de mes compagnes les plus "vieilles", que je trouvais tellement à plaindre, si bien qu'à travers elles je me trouvai aussi à plaindre. (EV, p. 45) 
Dans la conformité d'un rôle privilégié qui protège son autonomie et lui donne un pouvoir, dans une maternité sublimée qui n'exclut pas la tendresse, ce personnage féminin fait l'expérience d'une plénitude. ${ }^{17}$

Mais il n'en reste pas moins que Gabrielle Roy choisit de mettre en scène dans sa fiction une institutrice qui trouve son métier limitatif et contraignant et le quitte au profit d'une activité plus enrichissante. $\mathrm{Au}$-delà de la référence biographique joue la valeur symbolique d'un élément constitutif d'une vision du monde orientée vers la création.

Toujours dans la même optique, peut-être conviendrait-il d'interpréter le personnage maternel des trois récits plus spécifiquement reliés au choix de la carrière, soit "La voix des étangs" (RD), "Gagner ma vie" (RD) et "La route d'Altamont (RA), comme emblème de ce qui fait barrage à l'émancipation féminine. On voit alors apparaître l'autre face du personnage maternel, la face sombre et réductrice, qui encourage le statu quo. Dans "La voix des étangs", c'est bien le désir de la mère et non celui de la fille qui est exprimé dans le choix d'un métier:

Et elle me confia ce qu'elle désirait pour moi de toute son âme:

- Si tu voulais, Christine, devenir institutrice!... Il n'y a pas

d'occupation plus digne, il me semble, pour une femme... (RD, p. 283)

La fille avait plutôt songé à écrire mais, est-il dit, ne sachant comment gagner sa vie (RD, p. 282), elle s'emploierait à réaliser le rêve que ceux qui nous aiment font à notre usage (RD, p. 284). Devenir institutrice est donc bien le rêve de la mère qui continue de perpétuer les images stéréotypées, l'idée de ce qui est digne pour une femme. Implicitement; le texte établit une équation, aux yeux de la fille, entre gagner sa vie et la tâche de l'institutrice, tâche qui perd de la sorte sa valeur de vocation et qui se distingue nettement de cette occupation de tout l'être que serait l'écriture.' Parallèlement, c'est toujours avec réticence et appréhension que là mère reçoit les confidences de sa fille relatives au désir de devenir écrivaine, métier qui est ce qu'il y a de plus exigeant au monde (RD, p. 246) et qui éloigne d'autrui, qui voue à la solitude. La fille n'est pas encouragée à se mesurer aux épreuves et aux difficultés qui forgent l'être, mais elle est invitée à perpétuer passivement et en toute sécurité un rôle qui se situe dans le prolongement des valeurs maternelles traditionnelles. Dans «La route d'Altamont", l'acharnement que met Éveline (descendante d'Ėve ?) à contrer le projet de départ de sa fille vers le continent du savoir et de la culture, son désarroi et son dépéris-

17 Gabrielle Pascal, "La condition féminine dans l'œuvre de Gabrielle Roy », Voix \& images, vol. V, n⿳0 1 , automne 1979, p. 159. 
sement constituent encore des signes de mère périmée. L'éloignement dans l'espace, comme l'atteste le dialogue mère-fille qui suit, est la métaphore de la distanciation indispensable à l'écrivain pour recréer la réalité:

- [...] D'abord, si tu veux écrire, tu n'as pas besoin pour cela de courir au bout du monde. [...] Ici comme ailleurs, il y a à décrire la joie, les chagrins, les séparations...

- Mais pour le voir ne faut-il pas que je m'en éloigne? (RA, p. 238)

Et, dans le contexte, cet éloignement symbolise aussi une rupture avec un certain univers maternel. La disparition du personnage de la mère qui se produit avant le retour de la fille et qui clôt la nouvelle vient confirmer et comme accomplir cette rupture. Il fallait que meure la procréatrice pour qu'advienne la créatrice.

Le corps finit donc par se résorber dans la voix qui, fluide, légère, souveraine, assume l'œuvre de transmutation. Principale manifestation de l'ultime personnage féminin, celui de la narratrice-écrivaine, la voix, libérée et libératrice, va faire renaitre celle qui est morte de n'en avoir pas eu: [...] en vivant ma vie, c'est comme si je lui donnais une voix pour s'exprimer (RA, p. 227). Or on sait à quel point la voix porte en elle la question de l'origine et de la fusion avec la mère, ainsi que l'écrit René Payant dans un article intitulé "L'impossible voix ":

La voix conteuse de la mère relève la fonction bienfaisante du sein. L'oreille reçoit la voix comme une coulée chaleureuse et le plaisir de l'écoute se substitue à celui de la succion. Déjà, la musique de la voix s'adresse au corps entier qu'elle berce. ${ }^{18}$

Cette voix expressive, qui ne cesse de s'adresser directement au lecteur, renoue avec l'origine, ne retenant que la part créatrice de l'héritage maternel:

Elle m'avait enseigné le pouvoir des images, la merveille d'une chose révélée par un mot juste et tout l'amour que peut contenir une simple et belle phrase. (RD, p. 246)

Pareille reconnaissance de la filiation maternelle n'est pas sans rappeler une autre voix féminine célèbre, celle de Colette, qui se réclame elle aussi de la même source d'inspiration et de vie. Les mots de ma mère, dit-elle, ressurgissent maintenant de moi, et quelquefois on les trouve beaux ${ }^{19}$. Ou encore: Elle m'a donné le jour, et la mission de poursuivre ce qu'en poète elle saisit et abandonna, comme on s'empare d'un fragment de mélodie flottante, en voyage dans

18 René Payant, loc. cit.

19 Colette, la Naissance du jour, Paris, Flammarion, 1970, p. 41. 
l'espace... ${ }^{20}$ A quoi font écho à plusieurs années de distance, en

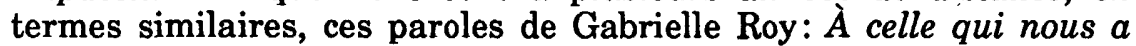
donné le jour, on donne naissance à notre tour quand, tôt ou tard, nous l'accueillons enfin dans notre moi. (RA, p. 226-227) Toujours situé au commencement, le Verbe est devenu maternel et vocalisé.

Il me parait déterminant que Gabrielle Roy situe l'origine de son métier d'écrivain dans l'audition du chant des grenouilles qui, invisibles, arrivent à échapper au fond gluant des marais grâce à leur mincè voix éclatante, sorte de musique aiguë, vibrante (RD, p. 243), cri de détresse ou cri triomphal (RD, p. 244). C'est à écouter ce cri troublant que la jeune Christine en vient à choisir la voie de l'écriture comme si elle recevait un ordre baroque: Ainsi, j'ai eu l'idée d'écrire. (RD, p. 244) En désignant la voix comme point d'origine de l'écriture, Gabrielle Roy se trouve à privilégier le spontané et le pulsionnel au détriment de la loi et du rationnel. C'est Anne Clancier qui fait remarquer avec beaucoup de pertinence qu'il

faut donner une place privilégiée à la voix dans la genèse de la création poétique. La voix est sans doute ce que l'enfant perçoit le plus précocement du monde extérieur, puisque même avant sa naissance, il peut entendre la voix de sa mère [...].:21

C'est à l'intérieur de la même nouvelle, "La voix des étangs", que s'exprime, en contradiction flagrante avec la voix des grenouilles, la profonde réticence maternelle à l'égard de l'écriture. La voix originelle qui émane de la nature n'a donc rien à voir avec la mère telle que la société l'a fabriquée. Elle a plutôt partie liée avec la mère originaire, hors culture. Cette opposition entre mère-loi et mère-voix ressurgit dans une nouvelle au titre significatif, "L'alouette" (EV, p. 41-59). De sa mère, Paraskavia Galaïda, femme simple, timide, non pervertie par la culture, Nil a hérité d'une voix pure d'alouette qui allège les cœurs lourds et soulage les âmes torturées. Grâce à ce don inné, "naturel ", l'enfant accomplit le miracle de faire marcher la vieille mère de la narratrice qu'un accident a immobilisée. Sous l'effet magique, inexplicable, de la voix aux inflexions tendres, captivantes, celle qui était prisonnière à la fois de son corps et de sa-passivité se remet en mouvement, retrouve la volonté de marcher. Il convient de noter que, comme pour le cri des grenouilles, c'est une voix sans paroles qui exerce sa fascination, voix qui touche par ses qualités sonores sans être occultée ou circonscrite par les limites des mots ou du sens. Car le petit Nil, comme sa mère, chante en ukrainien, langue étrangère pour ceux qui les écoutent. Guy Rosolato, qui situe

20 Ibid., p. 38.

21 Anne Clancier, "Le corps et ses images dans la création poétique", Corps création entre lettres et psychanalyse, publié sous la direction de Jean Guillaumin, Lyon, Presses universitaires de Lyon, 1980, p. 197. 
la voix entre corps et langage, souligne la part d'indéfinissable fascination qui s'attache à la voix sans égard à l'information transmise. En tant qu'elle ést la plus grande puissance d'émanation du corps, la voix, comme la musique vocale, possède ce trait majeur qui est

de pouvoir suggérer, remettre en circulation, d'une manière tout à fait physique, et à cause de cela, à cause de cette nécessité matérielle, d'atteindre plus sûrement le souvenir, le repère, mythique, des premières impositions du langage. ${ }^{22}$

Et ce sont bien les inflexions, les nuances, la musicalité de la voix de Nil, accompagnée de celle de sa mère, qui émeuvent les auditeurs:

Une délicate vibration musicale de la gorge fila un moment. Puis leurs voix partirent [...]. Alors elles montèrent et s'accordèrent en plein vol dans un chant étrangement beau qui était celui de la vie vécue et de la vie du rêve. (EV, p. 59)

Ces voix en plein vol se superposent à la métaphore récurrente de l'oiseau dans l'œuvre royenne pour revendiquer l'espace de la liberté et de la création. Expulsé par la bouche, projeté en plein ciel, le corps est devenu ce cœur-oiseau qu'un chant étrangement beau a libéré:

Sous le ciel immense, il [le chant] prenait le cour, le tournait et retournait, comme l'aurait fait une main, avant de le lâcher, pour un instant, avec ménagement, à l'air libre. (EV, p. 59)

22 Guy Rosolato, "La voix: entre corps et langage", Revue française de psychanalyse, vol. XXXVIII, $\mathrm{n}^{\circ} 1$, janvier 1974 , p. 76, 86. 